\title{
MAPPING ERODED AREAS ON MOUNTAIN GRASSLAND WITH TERRESTRIAL PHOTOGRAMMETRY AND OBJECT-BASED IMAGE ANALYSIS
}

\author{
Andreas Mayr ${ }^{\mathrm{a} *}$, Martin Rutzinger ${ }^{\mathrm{a}, \mathrm{b}}$, Magnus Bremer $^{\mathrm{a}}$ and Clemens Geitner ${ }^{\mathrm{a}}$ \\ ${ }^{\text {a }}$ Institute of Geography, University of Innsbruck, Innrain 52, 6020 Innsbruck, Austria \\ (andreas.mayr, martin.rutzinger, magnus.bremer, clemens.geitner)@uibk.ac.at \\ ${ }^{\mathrm{b}}$ Institute for Interdisciplinary Mountain Research, Austrian Academy of Sciences, Technikerstr. 21a, 6020 Innsbruck, Austria \\ martin.rutzinger@oeaw.ac.at
}

Commission V, WG V/5

KEY WORDS: Image Matching, OBIA, Erosion, Geomorphological Mapping, Thresholding, Excess Green Vegetation Index

\begin{abstract}
:
In the Alps as well as in other mountain regions steep grassland is frequently affected by shallow erosion. Often small landslides or snow movements displace the vegetation together with soil and/or unconsolidated material. This results in bare earth surface patches within the grass covered slope. Close-range and remote sensing techniques are promising for both mapping and monitoring these eroded areas. This is essential for a better geomorphological process understanding, to assess past and recent developments, and to plan mitigation measures. Recent developments in image matching techniques make it feasible to produce high resolution orthophotos and digital elevation models from terrestrial oblique images. In this paper we propose to delineate the boundary of eroded areas for selected scenes of a study area, using close-range photogrammetric data. Striving for an efficient, objective and reproducible workflow for this task, we developed an approach for automated classification of the scenes into the classes grass and eroded. We propose an object-based image analysis (OBIA) workflow which consists of image segmentation and automated threshold selection for classification using the Excess Green Vegetation Index (ExG). The automated workflow is tested with ten different scenes. Compared to a manual classification, grass and eroded areas are classified with an overall accuracy between $90.7 \%$ and $95.5 \%$, depending on the scene. The methods proved to be insensitive to differences in illumination of the scenes and greenness of the grass. The proposed workflow reduces user interaction and is transferable to other study areas. We conclude that close-range photogrammetry is a valuable low-cost tool for mapping this type of eroded areas in the field with a high level of detail and quality. In future, the output will be used as ground truth for an area-wide mapping of eroded areas in coarser resolution aerial orthophotos acquired at the same time.
\end{abstract}

\section{INTRODUCTION}

In the Alps as well as in other mountain regions shallow eroded areas occur frequently on steep grassland slopes from the montane to alpine elevation zone (Wiegand and Geitner, 2010). They are characterised by displacement of vegetation together with unconsolidated material. This type of eroded areas often results from small landslides or snow movements. Secondary erosion (e.g. by surface runoff) can deepen and expand the eroded areas. By mobilising and transporting material, these processes play a role for hillslope evolution. Depths exceed rarely more than a few decimetres and individual eroded areas typically range from 2 to $200 \mathrm{~m}^{2}$ (Tasser et al., 2005; Wiegand and Geitner, 2010). However, affected slopes typically feature many of these small areas (e.g. >30 areas per ha; Wiegand et al., 2013). The resulting loss of soil can have negative impacts on mountain agriculture and promote natural hazards. Thus, for example the soil conservation protocol of the Alpine Convention explicitly stipulates the monitoring and prevention of soil erosion (CIPRA, 2005).

An area-wide, yet accurate monitoring of eroded areas is required to gain a profound understanding of the erosion phenomenon and its dynamics. This is essential to evaluate current developments, estimate future trends and give recommendations on sustainable land management (Wiegand and Geitner, 2010, 2013; Meusburger and Alewell, 2014). Mapping in the field by global positioning system (GPS) surveys or manual digitalisation based on orthophoto interpretation, are often subjective and inaccurate. Moreover, given the small size and large number of the eroded areas, these approaches are unfeasible for large study areas and multitemporal mapping.

Today geomorphological mapping increasingly relies on automated techniques for classifying remotely sensed images and digital elevation models (DEMs) (Otto and Smith, 2013). Morphometric parameters, such as slope or curvature are derived to characterise geomorphological forms and processes. Arithmetic operations with image bands can highlight certain object classes. Vegetation indices are often used to classify vegetation and separate it from other classes (e.g. bare earth) in remote sensing data. The Excess Green Vegetation Index (ExG; Woebbecke et al., 1995) is one of the most popular indices if only bands in the visible light are available. Some studies apply object-based image analysis (OBIA) for geomorphologic classification of remote sensing data (e.g. Rau et al., 2014; Rutzinger et al., 2008; Schneevoigt et al., 2010; Kim et al., 2011). Compared to pixel-based classifications OBIA approaches often show superior performance with high resolution remote sensing data, particularly when pixels are smaller than the objects of interest. OBIA enables to combine

\footnotetext{
${ }^{*}$ Corresponding author
} 
image processing and spatial concepts (Blaschke, 2010). OBIA approaches are based on an initial segmentation of images or geometric data (e.g. 3D point clouds) into spatial entities called objects. Each object is analysed or assigned to a semantic class (classification) according to its characteristics in terms of size, shape, location, spectral properties or its spatial context (Bishop et al., 2012; Drăgut and Blaschke, 2006). A few recent studies (Torres-Sánches et al., 2015a, 2015b) combine automated thresholding of classification features and OBIA for vegetation classification in UAV (unmanned aerial vehicle) images. This strategy avoids the need for training areas in a classification procedure.

To map and analyse the spatio-temporal dynamics of shallow erosion Wiegand et al. (2013) used multi-temporal orthophotos. They used a range of thresholds for the classification of colour channels and compared the results to a classification with an expert-based threshold. Uncertainties in such a mapping approach result from limitations in the resolution and georeferencing of orthophotos as well as choice of the classification threshold. For a calibration and accuracy assessment of future classifications with updated orthophotos Wiegand et al. (2013) suggest collecting reference data in the field.

Reference data can be acquired for instance by measuring points at the boundary of eroded areas with a (differential) global positioning system. Modern close-range sensing methods (terrestrial laser scanning or photogrammetric image matching methods) can survey surfaces and geomorphological forms with a high level of detail and accuracy. Heng et al. (2010) and Rieke-Zapp and Nearing (2005) tested close-range photogrammetry to measure soil erosion (volumetric/elevation change) within a laboratory plot. Considering the type of erosion described in the beginning, we are at first interested in the areal extent of eroded surface. This is what can be extracted from remote sensing data with a larger spatial extent (Wiegand et al., 2013). Since we are about to develop and validate such area-wide approaches, we focus on this aspect here.

Geomorphological objects, however, often lack crisp boundaries. Instead, transitions are often fuzzy or gradient-like (Blaschke et al., 2014). This hampers an objective and repeatable survey of eroded areas with manual delineation in the field (e.g. with a differential GPS) or in close-range sensing data, since it is often unclear where to define and measure the boundary exactly. An automated delineation of eroded areas in close-range data is more objective but challenging as well. Again, the fuzziness of natural objects, as well as the noisiness of high resolution data representing natural surfaces hampers the delineation of discrete objects. Finally, the spectral characteristics of the classes vary with space and time (e.g. colour of grass depending on phenology, differences in illumination, colour of eroded areas depending on lithological differences of soil and substrate). This is compromising the robustness of a classification with a fixed threshold as well as its transferability to other study areas or even repeated measurements in the same area.

In this paper we propose to survey shallow eroded areas (mean size is about $30 \mathrm{~m}^{2}$ ) with a high level of detail and accuracy using close-range photogrammetry. Terrestrial oblique images of a hillslope scene affected by shallow erosion are processed with the image matching software Agisoft Photoscan (Agisoft, 2015) where Structure-from-Motion and dense matching algorithms are implemented (Snavely et al., 2006, 2008;
Fonstad et al., 2013; Westoby et al., 2012; Remondino et al., 2014). This produces 3D point clouds and centimetre resolution orthophotos (with $2 \mathrm{~cm}$ ground sample distance (GSD)). We present an automated approach to extract eroded (bare earth) and non-eroded (grass-covered) parts of each scene by objectbased image classification. Histogram based thresholding optimizes the classification with the Excess Green Vegetation Index $(E x G)$ in each scene without user intervention. This workflow is tested with 10 test plots / scenes and compared to a manual classification of the same orthophotos.

In upcoming projects this approach shall be used to acquire reference data (ground truth) for automated mapping of eroded areas in coarser scale remote sensing data (such as orthophotos acquired from unmanned or manned aircraft). This reference data is required for i) training the classifiers and ii) accuracy assessment. Furthermore, repeated surveys with this close-range sensing approach can be used for detailed change detection and plot to local scale monitoring of selected erosion hot spots.

In the following, we describe the acquisition of terrestrial images in the field and the production of orthophotos and point clouds from these images. Then, we propose an approach for segmentation and classification of eroded areas in an orthophoto scene. In Section 3 we show results for this classification in ten different scenes. We discuss these results in Section 4 and finally conclude our work in Section 5.

\section{METHODS}

\subsection{Data Acquisition}

The data used within this work was acquired at an east-facing, 30 - 40 degree steep slope, located below the summit of Hohe Warte (2398 m) in the Central Alps of Tyrol (Austria). Until the 1960s the slope was used as a meadow for hay making but today it is only used as a pasture for sheep. Ten different scenes on this slope were chosen to contain a bare earth surface patch where grass cover, soil and unconsolidated material had been eroded. Other unvegetated surface types, such as rock walls or scree slopes were avoided. Hence, the classification of each scene into eroded and non-eroded area (Section 2.4) corresponds basically to the task of separating grass covered (non-eroded) hillslope surface from eroded (bare earth) areas.

For each scene a set of several hundred oblique images was taken. We mounted the camera, a Canon $5 \mathrm{D}$, on a pole to elevate it higher above the ground. The shutter was released with a cable remote control. The images were acquired from multiple positions in an approximately circular pattern around the eroded area, with a focal length of $24 \mathrm{~mm}$. For use as ground control points (GCPs) four polystyrene spheres (with a $10 \mathrm{~cm}$ radius) were pinned to the ground with $300 \mathrm{~mm}$ nails. Their positions were surveyed with a real-time kinematic differential Global Positioning System (RTK-DGPS).

\subsection{Image Matching}

Three-dimensional (3D) models of the scenes were reconstructed from the image sets in Agisoft Photoscan 1.1.6 (Agisoft, 2015). This software implements the Structure-fromMotion (SfM) approach (Snavely et al., 2006, 2008; Fonstad et al., 2013) and dense matching (Remondino et al., 2014). The GCPs were used to georeference the models. 
The accuracy of the GCPs is crucial for the accuracy of the models since they were used for georeferencing (and thus scaling). The precisions of the DGPS survey were reported to be in the order of $1-2 \mathrm{~cm}$. According to the provider of the GPS correction data, accuracies of $\pm 1.5 \mathrm{~cm}$ horizontally and $\pm 4 \mathrm{~cm}$ vertically (mean error with a confidence level of approximately $68 \%$ ) can be assumed for the corrected positions under good conditions (Federal Office of Metrology and Surveying, 2015). Moreover, inaccuracies are introduced by local conditions which are not corrected with the differential technique as well as by placing the spheres and the antenna. Thus, we estimate the accuracies of the GCPs to be in the order of $10 \mathrm{~cm}$.

Moreover, non-linear distortions of the 3D model can cause inaccuracies (Fonstad et al., 2013). These can result from the SfM based reconstruction of a sparse point cloud and estimation of the camera parameters using convergent terrestrial oblique images. We tried to reduce such inaccuracies by including the GCPs in the bundle adjustment. Moreover, we assume the image block to be fairly stable due to the large number of images with high overlap.

The 3D models were textured to produce orthophotos with $2 \mathrm{~cm}$ resolution by mosaicking the image set. 3D point clouds were aggregated to a raster digital elevation model (DEM, cell size $2 \mathrm{~cm}$ ), using the $25 \%$ trimmed mean of the points' $z$-values in each raster cell (to discard outliers). The orthophotos, DEMS and point clouds were manually cut to exclude peripheral parts of each scene where the quality of the image matching is poor.

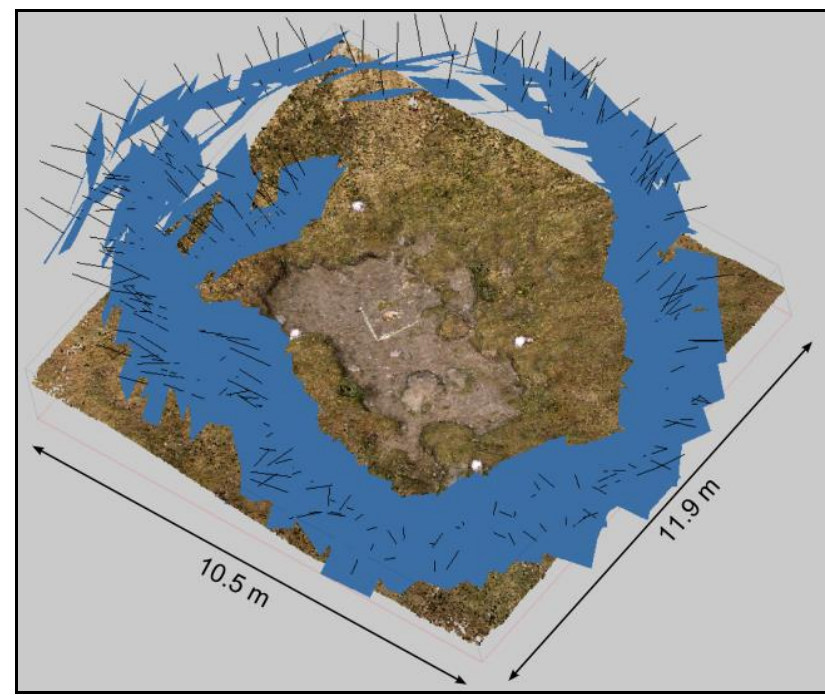

Figure 1: RGB coloured 3D point cloud of scene 10. Blue planes and black lines represent the reconstructed camera positions and orientations.

\subsection{Segmentation}

Segmentation and the subsequent classification workflow are implemented with Python scripts (Python Software Foundation, 2015) and SAGA GIS (Conrad et al., 2015). Each scene is segmented by seeded region growing (Adams and Bischof, 1994) based on four raster features: the RGB (red, green, blue) channels of the orthophoto and slope. The morphometric attribute slope is used to constrain the region growing at edges of the eroded area where the spectral features may be influenced by shadow. Slope is derived from the raster DEM with a window size of $5 \times 5$ cells using the method proposed by Wood
(1996). Seed points are generated as the minima of variance of the normalized features (R, G, B and slope) using a bandwidth of 15 cells (Adams and Bischof, 1994).

\subsection{Vegetation Index and Classification}

2.4.1 Excess Green Vegetation Index (ExG): In order to classify grass and eroded area we calculate the Excess Green Vegetation Index (ExG; Woebbecke et al., 1995) from the red $(\mathrm{R})$, green $(\mathrm{G})$ and blue (B) colour bands of the orthophotos, with

$$
E x G=2 g-r-b
$$

and

$$
\begin{aligned}
& g=G /(R+G+B) \\
& r=R /(R+G+B) \\
& b=B /(R+G+B) .
\end{aligned}
$$

2.4.2 Classification threshold: The data was acquired on several days between July and late September. Along with the phenological cycle of the grass, its colour changed from green towards brown. Moreover, differences in the spectral feature space result from varying illumination as well as variability of the bare earth properties (grain sizes of the material, soil vs. rock). An automated thresholding for the classification with spectral features is implemented to adapt to the characteristics of each scene individually. This will allow batch processing of a large number of scenes without the need for manual threshold selection or a supervised classification with training areas.

Despite the brownish colour of the grass in some scenes the ExG values still tend to be higher in the grass covered part of the scene than in the eroded area. Thus, for the thresholding we assume a bimodal frequency distribution (histogram) of the ExG values, with each class forming one peak. In order to enhance the separability of the two classes grass and eroded a Gaussian filter is applied to blur the ExG raster. The parameters standard deviation $\sigma=1$ and search radius $r=2$ are used. This processing step has advantages for the subsequent determination of the classification threshold because it smoothes the ExG histogram and pronounces its bimodality. For the binarization of images with bimodal histograms Otsu's method is frequently used (Otsu, 1979). This method, however, has problems if the two clusters in the histogram differ in size. In such cases our initial tests found the threshold to be biased towards the larger cluster. Therefore, we apply a different technique to optimize the threshold. The ExG histogram is analysed to find the two peaks first. Subsequently, the minimum between the peaks is searched and used as the classification threshold.

2.4.3 Object-Based Classification: The raster based feature ExG is aggregated to the segments as mean values per segment. The resulting segment feature $E_{x G_{\text {mean }}}$ characterizes the segments (objects) in terms of spectral properties. With the classification threshold determined from the ExG raster histogram the segments are classified as grass or eroded depending on their $\operatorname{ExG}_{\text {mean }}$ value. Finally, the classification output can be vectorised for further analysis. 


\section{RESULTS}

\subsection{Image Matching}

Table 1 summarizes the SfM-based reconstruction of the 10 scenes. This overview is intended to evaluate the quality of the image matching. The SfM procedure was able to align between $68 \%$ and nearly $100 \%$ of the images that were used as input. This resulted in 36,000 - 110,000 tie points per scene (sparse point cloud). Marker errors (i.e. mean deviations of GCPs in the model from GPS coordinates of the GCPs) typically are in the order of $2-3 \mathrm{~cm}$. For two eroded areas, however, they are reported to be considerably higher $(8 \mathrm{~cm}$ and $16 \mathrm{~cm})$. The final dense point clouds contain between 1.5 and 5.2 Mio points. The point clouds appear smoothed to a certain degree but still contain morphological information concerning the more prominent structures. Edges at eroded area scarps and clods of soil and grass within the eroded areas are morphologically well represented (Figure 2). Most of the reconstructed GCP spheres appear at least with a sphere-like shape although they sometimes show some deformation or noise. A folding rule (scale bar) of $2 \mathrm{~m}$ length was placed in all scenes except scene 6 (with its two $1 \mathrm{~m}$-segments directed orthogonally; see Figure 4). By measuring the segments' 3D length in the point cloud a scale error is determined for each reconstructed scene (maximum deviation of the two segments from reference; see Table 1). The point density is generally high (typically several hundred points per $\mathrm{dm}^{2}$ ) within the area of interest of a scene (i.e. the eroded area and its direct surroundings). Areas with lower point density occur at steep scarps of the eroded areas if they are in shadow due to their aspect relative to the sun position. Peripheral parts of the scenes suffer from considerably lower image matching quality. This was expected due to reduced coverage/overlap of images as well as increased ranges and obliquity of the images for these parts.

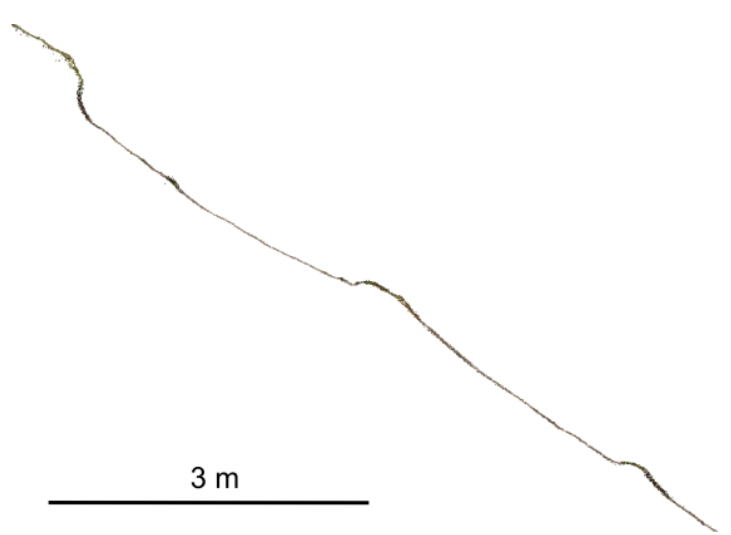

Figure 2: Cross-section through a point cloud visualized in Cloud Compare (Girardeau-Montaut, 2011). The eroded area scarp (upper left) and two clods of soil within the eroded area (middle and lower right) can be identified.

The orthophotos ( $2 \mathrm{~cm}$ GSD) contain a high level of detail but also a relatively high degree of noise. Only locally, the orthophotos appear very blurred, particularly in peripheral parts of a scene (where images were poorly matched; see above).

The image matching results for scene 5 are the poorest of the entire data set (Table 1). Only $68 \%$ of the 342 images were aligned, resulting in a relatively low number of tie points $(39,828)$. Additionally, the markers errors $(16 \mathrm{~cm})$ and scale errors $(6 \%)$ were the highest in this scene. The orthophoto shows blur in some parts and the spheres are not reconstructed correctly.

\begin{tabular}{cccrcc}
\hline Scene & $\begin{array}{c}\text { Images } \\
\text { used }\end{array}$ & $\begin{array}{c}\text { Images } \\
\text { aligned }\end{array}$ & \multicolumn{1}{c}{$\begin{array}{c}\text { Tie } \\
\text { points }\end{array}$} & $\begin{array}{c}\text { Marker } \\
\text { errors [m] }\end{array}$ & $\begin{array}{c}\text { Scale } \\
\text { error [\%] }\end{array}$ \\
\hline $\mathbf{1}$ & 613 & 546 & 106,000 & 0.03 & 1 \\
$\mathbf{2}$ & 539 & 484 & 55,000 & 0.03 & 2 \\
$\mathbf{3}$ & 676 & 505 & 110,214 & 0.01 & 3 \\
$\mathbf{4}$ & 480 & 342 & 77,315 & 0.08 & 1 \\
$\mathbf{5}$ & 342 & 234 & 39,828 & 0.16 & 6 \\
$\mathbf{6}$ & 243 & 233 & 47,000 & 0.04 & - \\
$\mathbf{7}$ & 179 & 143 & 36,000 & 0.02 & 1 \\
$\mathbf{8}$ & 279 & 275 & 60,787 & 0.03 & 1 \\
$\mathbf{9}$ & 406 & 399 & 99,000 & 0.03 & 2 \\
$\mathbf{1 0}$ & 251 & 250 & 53,000 & 0.03 & 2 \\
\hline
\end{tabular}

Table 1: Number of images that were used as input, number of images that could be aligned by SfM, number of tie points, marker errors and scale errors.

\subsection{Segmentation}

Segments produced for a scene by seeded region growing are shown in Figure 3. The parameters used for seed point generation (Section 2.3) created enough seed points to grow relatively small segments, compared to the objects of interest (eroded areas). This reduces the risk of a segment containing both grass and eroded area.

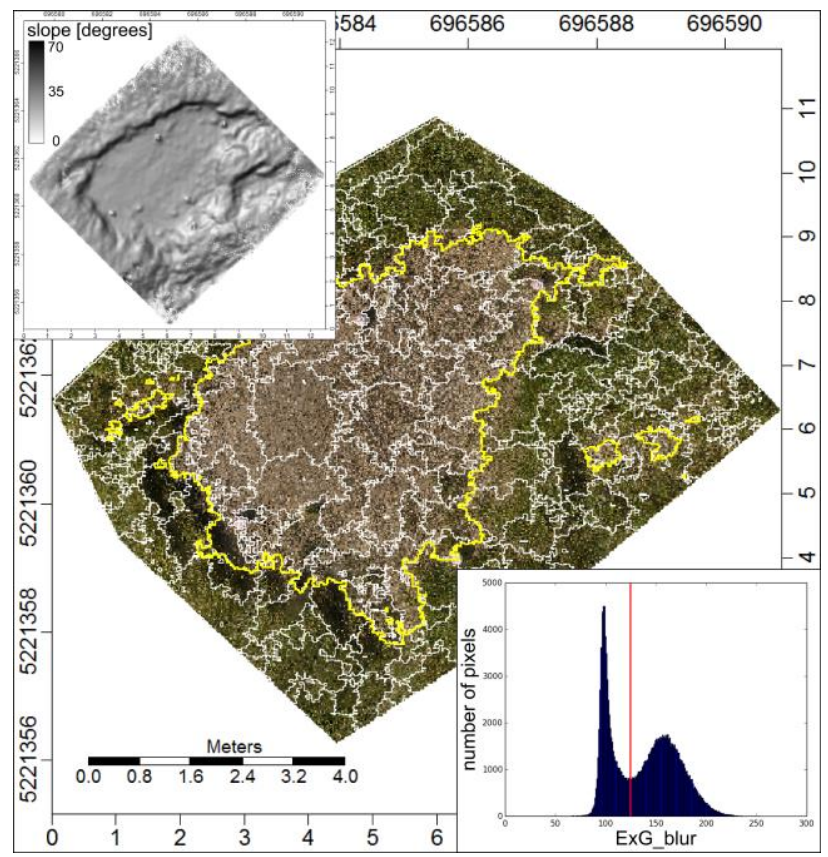

Figure 3: Results of the segmentation (white) and classification (eroded area boundaries = yellow) for scene 3 . The slope map (upper left) is used together with the RGB channels to segment the scene. The histogram for the Gaussian filtered ExG (lower right) is used to define the classification threshold (red line). 


\subsection{Classification}

Most of the classified scenes contain one main eroded area and sometimes a couple of small ones. In the following these multipart objects of a scene are analysed as one object. For any further analysis steps they could be vectorised and filtered by size to exclude very small objects. Table 2 presents the sizes of the classified eroded areas in the ten scenes. Their mean size is about $30 \mathrm{~m}^{2}$. The smallest area is $4 \mathrm{~m}^{2}$ and the largest area is 51 $\mathrm{m}^{2}$. In order to assess the results of the automated classification they are compared to a manual classification of the ten scenes based on the same orthophotos (Table 2). For most scenes the error of the determined size (i.e. the deviation from the manual classification) is below $10 \%$. For the scenes 5 and 8 , however, the size is overestimated by $18 \%$ and underestimated by $14 \%$ respectively.

\begin{tabular}{crrrr}
\hline Scene & $\begin{array}{r}\text { Eroded area } \\
\text { automated }\left[\mathbf{m}^{2}\right]\end{array}$ & $\begin{array}{r}\text { Eroded area } \\
\text { manual }\left[\mathbf{m}^{2}\right]\end{array}$ & $\begin{array}{r}\text { Error } \\
{[\mathbf{\%}]}\end{array}$ & $\begin{array}{r}\text { Error } \\
{\left[\mathbf{m}^{2}\right]}\end{array}$ \\
\hline $\mathbf{1}$ & 51.4 & 50.7 & 1.5 & 0.8 \\
$\mathbf{2}$ & 28.9 & 31.0 & -6.8 & -2.1 \\
$\mathbf{3}$ & 22.4 & 24.6 & -8.8 & -2.2 \\
$\mathbf{4}$ & 37.4 & 38.5 & -2.9 & -1.1 \\
$\mathbf{5}$ & 37.7 & 31.8 & 18.4 & 5.9 \\
$\mathbf{6}$ & 4.0 & 4.3 & -5.4 & -0.2 \\
$\mathbf{7}$ & 15.0 & 15.3 & -1.6 & -0.3 \\
$\mathbf{8}$ & 29.7 & 34.5 & -13.9 & -4.8 \\
$\mathbf{9}$ & 59.4 & 57.0 & 4.2 & 2.4 \\
$\mathbf{1 0}$ & 12.0 & 13.2 & -9.2 & -1.2 \\
\hline
\end{tabular}

Table 2: Sizes of the eroded areas classified in the ten scenes with the automated approach and manually. The error of the automated classification is calculated as the deviation from the manual classification.

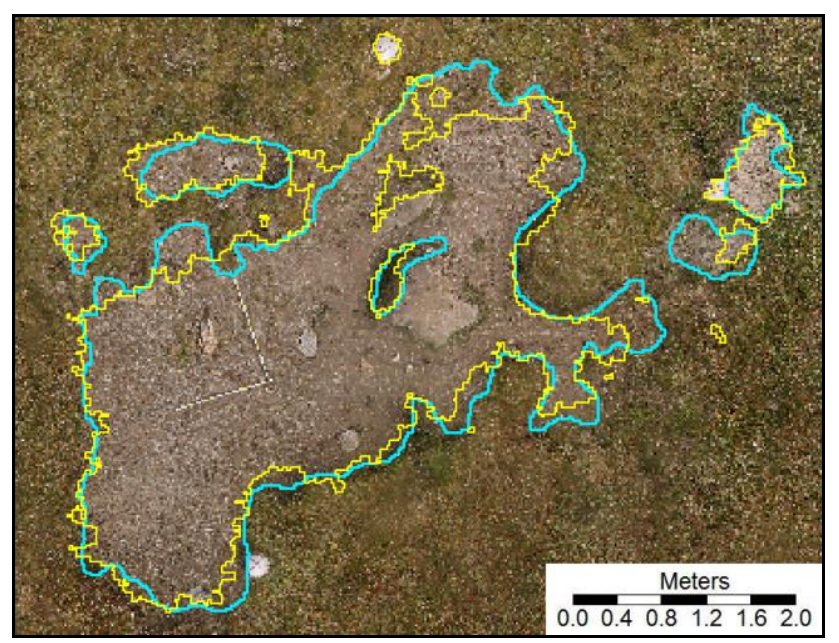

Figure 4: Classification results (eroded area boundaries) for scene 10 (yellow) and the manual classification (blue).

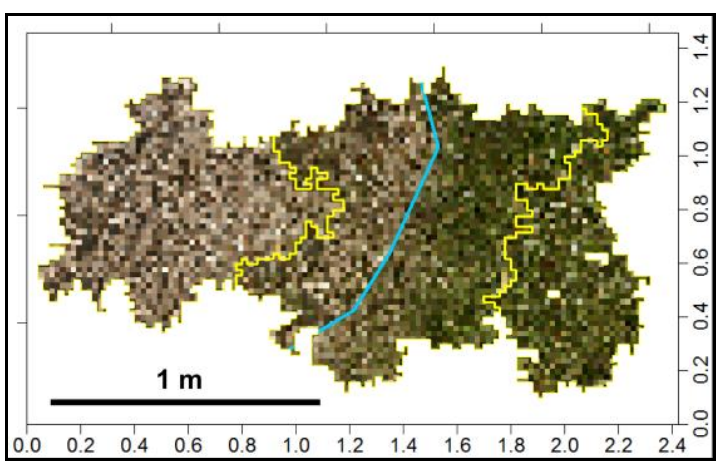

Figure 5: Gradient-like transition from eroded to grass. The segment in the middle (yellow boundaries) covers the transition zone. Thus, classification of this segment will result in error, compared to the manual classification (blue).

For each scene individually as well as for all scenes together, the producer's and user's accuracies of the two classes (grass and eroded) as well as the overall accuracy are calculated (Table 3; cf. Congalton, 1991). Compared to a manual mapping, grass and eroded areas are classified with an overall accuracy between $90.7 \%$ and $95.5 \%$, depending on the scene.

\begin{tabular}{|c|c|c|c|c|}
\hline Scene & Class & $\begin{array}{c}\text { User's } \\
\text { accuracy } \\
{[\%]}\end{array}$ & $\begin{array}{c}\text { Producer's } \\
\text { accuracy } \\
{[\%]}\end{array}$ & $\begin{array}{c}\text { Overall } \\
\text { accuracy } \\
{[\%]}\end{array}$ \\
\hline 1 & eroded & 95.7 & 94.3 & \multirow{2}{*}{95.4} \\
\hline 1 & grass & 95.2 & 96.4 & \\
\hline 2 & eroded & 90.7 & 97.3 & \multirow{2}{*}{95.4} \\
\hline 2 & grass & 98.4 & 94.3 & \\
\hline 3 & eroded & 86.7 & 95.0 & \multirow{2}{*}{92.8} \\
\hline 3 & grass & 96.9 & 91.5 & \\
\hline 4 & eroded & 90.8 & 93.5 & \multirow{2}{*}{94.5} \\
\hline 4 & grass & 96.6 & 95.1 & \\
\hline 5 & eroded & 94.0 & 79.4 & \multirow{2}{*}{90.7} \\
\hline 5 & grass & 89.2 & 97.1 & \\
\hline 6 & eroded & 82.9 & 87.6 & \multirow{2}{*}{94.3} \\
\hline 6 & grass & 97.1 & 95.8 & \\
\hline 7 & eroded & 87.7 & 89.1 & \multirow{2}{*}{92.6} \\
\hline 7 & grass & 94.9 & 94.2 & \\
\hline 8 & eroded & 83.0 & 96.4 & \multirow{2}{*}{94.3} \\
\hline 8 & grass & 98.8 & 93.6 & \\
\hline 9 & eroded & 93.8 & 90.0 & \multirow{2}{*}{93.3} \\
\hline 9 & grass & 93.1 & 95.7 & \\
\hline 10 & eroded & 83.6 & 92.1 & \multirow{2}{*}{95.5} \\
\hline 10 & grass & 98.3 & 96.2 & \\
\hline all & eroded & 90.7 & 91.0 & \multirow{2}{*}{94.3} \\
\hline all & grass & 95.9 & 95.8 & \\
\hline
\end{tabular}

Table 3: Accuracy of the classification results compared to a manual classification of eroded areas and grass with the same data. Colour ramp: red (low accuracy) - yellow - green (high accuracy). 


\section{DISCUSSION}

In the following, we discuss our results from the ten scenes with regard to their accuracy and limitations of the approach that are identified by our tests. Moreover, we suggest how the remaining challenges may be tackled in future work.

As expected, the orthophotos (with a GSD of $2 \mathrm{~cm}$ ) produced by matching of the close-range images show much more detail than aerial orthophotos of the study area $(\mathrm{GSD}=20 \mathrm{~cm})$. Low point cloud densities at steep scarps of the eroded areas probably result from shadow due to their aspect relative to the sun position. Peripheral parts of the scenes suffer from considerably lower image matching quality. This was expected due to reduced coverage/overlap of images as well as increased ranges and obliquity of the images for these parts. Bearing this in mind, the orthophotos and point clouds from terrestrial close range photogrammetry have a high potential for local scale geomorphological investigations.

Problems with image alignment (e.g. in scene 5) seem to occur particularly when camera positions are not distributed well but if they are rather clustered at a few standpoints. If these points are too distant or image overlap is insufficient the SfM algorithm probably fails to find corresponding features in the images and thus cannot reconstruct the camera positions. This causes gaps in the reconstructed circle-like camera position pattern. Hence, it is important to acquire well-directed images from well-distributed positions. In practice, this can be difficult in steep terrain. Therefore, we took a large number of images to increase the chance of aligned images with good coverage of the scene.

With regard to the classification, we note that we did not exclude the white GCP spheres from the orthophotos. The workflow is not designed to classify them systematically. Accordingly, their classification as either grass or eroded is inconsistent. This means they account for some (unsystematic) error. For future studies at a similar scale we recommend smaller spheres (e.g. tennis balls) as they will be sufficiently visible in the images. This reduces both their bias on the classification and the volume of the field equipment.

Taken all scenes together, both user's and producer's accuracy for eroded are slightly lower than for grass. This means that the approach tends to underestimate the class eroded slightly. For the ten scenes the mean user's and the mean producer's accuracy are $92.4 \%$ and $93.2 \%$ respectively, with standard deviations of $5.1 \%$ and $4.1 \%$. This indicates that the classification accuracy is relatively constant from scene to scene. Thus, it can be assumed that the approach will deliver results with comparable accuracies $(92.4 \% \pm 5.1 \%$ and 93.2 $\pm 4.1 \%$ ) when applied to other scenes under similar conditions.

The overall accuracy for scene 1 is the second highest of all scenes. Producer's and user's accuracy are well balanced for both eroded and grass. This indicates a good classification success. That was expected because the images for this scene were acquired in July, when the grass was greener than during the other field campaigns in September. The contrast of the eroded area to the surrounding grass is better than for scenes with brownish grass. This has advantages for both the segmentation and the classification steps of the workflow.

According to our tests the approach works well in the inner part of an eroded area, provided that there is no or only little occurrence of vegetation (Vegetation can occur inside an eroded area due to natural succession of vegetation or clods of soil and grass being transported and deposited.). Ideally, the boundary is well defined by a relatively sharp edge with good illumination (i.e. homogeneous illumination due to cloudy conditions or the position of the sun did not produce shadows in the eroded area). In these cases the classification is most accurate and robust. Misclassifications of some segments occur close to scarps that are casting shadows in the eroded area. In this regard, the integration of $(2.5 \mathrm{D} / 3 \mathrm{D})$ geometric information (morphometric parameters, such as surface roughness) in the information extraction approach may improve the classification results. Parts of an eroded area where vegetation regrowth/succession has already occurred are problematic as well. Including segment features that e.g. describe the texture or the variability of spectral values within a segment may provide an opportunity to classify an additional class (regrown). This would add value to the classification from a process-oriented geomorphological or ecological point of view. In some scenes clods of material covered by grass lie in the eroded areas (e.g. scene 10; Figure 4). They either have resisted the erosion processes or they have been moved from above (by gravitation or snow movement) and deposited inside the eroded area. This raises the question what to do with grass segments representing such objects. One possibility is to include them into the surrounding eroded area object using object-based analysis and topological rules.

Some scenes show relatively low user's accuracies for eroded. Scene 8 , for instance, has only a user's accuracy of $83.0 \%$. This means that $83 \%$ of the pixels in scene 8 that are classified as eroded by the automated approach are also eroded according to the manual classification. A closer look at this scene reveals that the differences between automated and manual classification are due to a combination of the problems described in the previous paragraph (shadows, gradual transitions and vegetation inside the eroded area).

The overall accuracy for scene 5 is the lowest. The producer's accuracy for eroded is only $79.4 \%$ due to a relatively high number of false negatives. This may be related to the fact that the image matching results for this scene are also the poorest of the entire data set (see Table 1). On the other hand, the original images show that the captured eroded area lacks a crisp boundary in downslope direction. In terms of vegetation abundance, and thus greenness, the transition from eroded area to grass is gradient-like. Morphologically as well, the transition is rather smooth than a pronounced edge. These conditions are unfavourable for a reliable and accurate mapping of the eroded area as a discrete object, both with a manual classification and with the automated approach.

Figure 5 shows a situation in scene 3 that is problematic for the approach. One segment covers a gradient-like transition zone from eroded area to grass. This highlights the problem that a discrete classification to one of the two classes cannot represent the fuzziness of an eroded area. To address this aspect Wiegand et al. (2013) implemented the concept of vague objects (Dilo, 2006) in a pixel-based classification of eroded areas with varying thresholds. Such approaches may be appropriate to characterize and define natural objects more realistically.

In addition to the classification accuracy, the absolute accuracy of the delineated eroded areas depends on the spatial accuracy of the orthophoto produced by close-range image matching. This in turn is depending on the quality of the image matching 
and on the accuracy of the GCPs. Considering our estimate of GCP accuracy and the marker errors from image matching (Section 2.2), the spatial accuracy of the close-range photogrammetric orthophoto and point cloud is estimated to be in the order of $10 \mathrm{~cm}$. This can be regarded as sufficient for the intended purpose of eroded area mapping, in particular if the close-range mapping results are to be used as ground truth for a mapping with coarser resolution and less accurate aerial orthophotos. In future work, a comparison of a photogrammetric point cloud and a point cloud from terrestrial laser scanning may provide a validation of the image matching accuracy under the given conditions (natural surfaces, camera configuration and image acquisition geometry). For a change detection with repeated close-range surveys the classification accuracy as well as the positional and geometric accuracy of the data must be considered. These have implications on the minimum size of detectable changes.

\section{CONCLUSIONS}

We presented an application of close-range photogrammetry in geomorphological mapping. Our work comprises the entire workflow from data acquisition in the field to automated information extraction from the photogrammetric data. For our study, close-range photogrammetry had advantages over terrestrial laser scanning because of the relatively lightweight equipment and the lack of suitable scanning positions with a good view of the scenes. An advantage of the OBIA approach, compared to a pixel-based classification, is that it copes with the noise of the high resolution data. Smoothing of the spatial data is avoided prior to segmentation of the image to keep the eroded area boundaries as crisp and spatially accurate as possible. Aggregation of features on a segment basis (averaging pixel-based features over homogeneous objects), however, avoids a salt-and-pepper effect in the final classification. Avoiding the need for manual threshold selection or training areas, the information extraction workflow has advantages in terms of repeatability, objectiveness and transferability to other study areas. The workflow was successfully applied to ten scenes in the study area. The scenes are characterised by different conditions in terms of illumination and colour of the vegetation. Compared to a manual classification in the photogrammetric data, grass and eroded areas are classified with an overall accuracy of $94.3 \%$. It is noted that the manual classification (as well as a GPS survey) of eroded area boundaries is not necessarily more accurate. The visual interpretation and definition of (in reality often fuzzy and gradual) boundaries by a surveyor involves a certain amount of subjectivity and hence the potential for inconsistency. Hence, we conclude that the automated and the manual approach are in relatively good accordance while the automated approach has advantages in terms of objectiveness and repeatability. The accuracy analysis highlights the importance of the image matching quality for the subsequent classification. Consequently, enough time should be reserved for a thorough and well-directed image acquisition in the field. We recommend acquiring several hundred images per scene with high overlap and from well distributed positions. This increases the redundancy and the stability of the image block geometry during reconstruction of the scene by image matching. Additionally, a strong focus should be on the accuracy of the GCPs. Precision and accuracy may be improved by additional, well distributed GCPs. If a larger areal coverage is the objective the image acquisition from an unmanned aerial vehicle (UAV) is an interesting option. Moreover, the results show that the fuzziness of many natural objects, such as eroded areas, still is a challenge for information extraction and delineation of discrete objects. However, we can conclude for future work that erosion areas mapped by terrestrial close-range photogrammetry are a reliable ground truth for the coarser resolution and less accurate aerial orthophotos.

This study investigated the general applicability of automated image matching for eroded area mapping, including the information extraction part of the workflow. Future methodological work may focus on a more rigorous accuracy assessment of the orthophotos and point clouds or of the final outputs (i.e. delineated eroded areas). The presented approach may also be interesting for landslide mapping. Though, due to the larger size of most landslides, a different image acquisition strategy may be necessary in order to capture the entire landslide. In this case image acquisition from the opposite slope or from a UAV will be more appropriate.

\section{ACKNOWLEDGEMENTS}

This work was funded by the Mountain Agricultural Research Unit (University of Innsbruck / Federal State of Tyrol) and by a fellowship of the Vice Rectorate for Research of the University of Innsbruck (Austria)

\section{REFERENCES}

Adams, R., \& Bischof, L., 1994. Seeded region growing. IEEE Transactions on Pattern Analysis and Machine Intelligence, 16(6), 641-647.

Agisoft, 2015. Agisoft PhotoScan User Manual: Professional Edition, Version 1.2. http://www.agisoft.com/pdf/photoscanpro_1_2_en.pdf (09.12.2015).

Bishop, M. P., James, L. A., Shroder, J. F., \& Walsh, S. J., 2012. Geospatial technologies and digital geomorphological mapping: Concepts, issues and research. Geomorphology, 137(1), 5-26.

Blaschke, T., 2010. Object based image analysis for remote sensing, ISPRS Journal of photogrammetry and remote sensing, $65,2-16$

Blaschke, T., Hay, G. J., Kelly, M., Lang, S., Hofmann, P., Addink, E., et al. (2014). Geographic Object-Based Image Analysis - Towards a new paradigm. ISPRS Journal of Photogrammetry and Remote Sensing, 87, 180-191

CIPRA - Commission Internationale pour la Protection des Alpes, 2005. Protocol on the implementation of the Alpine Convention of 1991 in the field of soil conservation. Official Journal of the European Union, 337/29

Congalton, R. G., 1991. A review of assessing the accuracy of classifications of remotely sensed data. Remote sensing of environment, 37(1), 35-46.

Conrad, O., Bechtel, B., Bock, M., Dietrich, H., Fischer, E., Gerlitz, L., Wehberg, J., Wichmann, V., and Boehner, J., 2015. System for Automated Geoscientific Analyses (SAGA) v. 2.1.4. Geoscientific Model Development, 8, 1991-2007. 
Dilo, A., 2006. Representation of and reasoning with vagueness in spatial information: A system for handling vague objects. Ph.D. thesis, University of Twente.

Drăgut, L., \& Blaschke, T., 2006. Automated classification of landform elements using object-based image analysis. Geomorphology, 81(3), 330-344

Federal Office of Metrology and Surveying (Bundesamt für Eich- und Vermessungswesen), 2013. APOS - Austrian Positioning Service. http://www.bev.gv.at (12.12.2015).

Fonstad, M. A., Dietrich, J. T., Courville, B. C., Jensen, J. L., \& Carbonneau, P. E. (2013). Topographic structure from motion: a new development in photogrammetric measurement. Earth Surface Processes and Landforms, 38(4), 421-430.

Girardeau-Montaut, D., 2011: Cloudcompare, a 3D point cloud and mesh processing free software. Tech. rep., EDF Research and Development, Telecom ParisTech.

http://www.danielgm.net/cc/.

Heng, P., B. C., Chandler, J. H., \& Armstrong, A., 2010. Applying close range digital photogrammetry in soil erosion studies. The Photogrammetric Record, 25(131), 240-265.

Kim, M., Warner, T. A., Madden, M. \& Atkinson, D. S., 2011. Multi-scale GEOBIA with very high spatial resolution digital aerial imagery: scale, texture and image objects, International Journal of Remote Sensing, 32, 2825-2850

Meusburger, K., \& Alewell, C., 2014. Soil Erosion in the Alps. Experience gained from case studies (2006-2013). Federal Office for the Environment, Bern. Environmental Studies, 1408.

Otsu, N., 1979: A threshold selection method from gray-level histogram. IEEE Transactions on Systems, Man, and Cybernetics, 9, 62-66.

Otto, J. \& Smith, M., 2013. Chap. 2.6 Geomorphological mapping, In: Clarke, L.E. (Ed.): Geomorphological Techniques (Online Edition), British Society for Geomorphology

Python Software Foundation, 2015. Python Language Reference, version 2.7. Available at http://www.python.org

Rau, J.-Y., Jhan, J.-P., \& Rau, R.-J., 2014. Semiautomatic Object-Oriented Landslide Recognition Scheme From Multisensor Optical Imagery and DEM. Geoscience and Remote Sensing, IEEE Transactions on, 52(2), 1336-1349.

Remondino, F., Spera, M. G., Nocerino, E., Menna, F., \& Nex, F., 2014. State of the art in high density image matching. The Photogrammetric Record, 29(146), 144-166.

Rieke-Zapp, D. H., \& Nearing, M. A., 2005. Digital close range photogrammetry for measurement of soil erosion. The Photogrammetric Record, 20(109), 69-87.

Rutzinger, M., Höfle, B., \& Pfeifer, N., 2008. Object detection in airborne laser scanning data-an integrative approach on object-based image and point cloud analysis. In: Blaschke et al. (Eds): Object-Based Image Analysis, 645-662.

Schneevoigt, N., der, S. v., Kellenberger, T., Kääb, A., \& Schrott, L., 2010. Object-oriented classification of alpine landforms from an ASTER scene and digital elevation data
(Reintal, Bavarian Alps). In: 10th International Symposium on High Mountain Remote Sensing Cartography (pp. 53-62).

Snavely N, Seitz SM, Szeliski R., 2006. Photo tourism: exploring photo collections in 3D. ACM Transactions on Graphics, 25 (3), 835 - 846.

Snavely N, Seitz SM, Szeliski R., 2008. Modeling the world from internet photo collections. International Journal of Computer Vision, 80 (12), 189-210.

Tasser, E., Mader, M., \& Tappeiner, U., 2005. Auswirkungen von Bewirtschaftungsänderungen auf die Blaikenbildung im Gebirge. Mitteilungen der Österreichischen Bodenkundlichen Gesellschaft, 72, 25

Torres-Sánchez, J., López-Granados, F., Serrano, N., Arquero, O., \& Peña, J. M., 2015a. High-throughput 3-D monitoring of agricultural-tree plantations with unmanned aerial vehicle (UAV) technology. PloS one, 10(6).

Torres-Sánchez, J., F. López-Granados, J.M. Peña, 2015b. An automatic object-based method for optimal thresholding in UAV images: Application for vegetation detection in herbaceous crops, Computers and Electronics in Agriculture, $114,43-52$

Westoby, M., Brasington, J., Glasser, N., Hambrey, M. \& Reynolds, J., 2012. 'Structure-from-Motion' photogrammetry: A low-cost, effective tool for geoscience applications. Geomorphology, 179, 300-314

Wiegand, C. \& C. Geitner, 2010. Shallow erosion in grassland areas in the Alps. What we know and what we need to investigate further. - In: Borsdorf, A., Grabherr, G., Heinrich, K., Scott, B. und J. Stötter (Hrsg.): Challenges for mountain regions - tackling complexity, Innsbruck, Wien: 76-83.

Wiegand, C., \& Geitner, C., 2013. Investigations into the distribution and diversity of shallow eroded areas on steep grasslands in Tyrol (Austria). Erdkunde, 67(4), 325-343

Wiegand, C., Rutzinger, M., Heinrich, K., \& Geitner, C., 2013. Automated extraction of shallow erosion areas based on multitemporal ortho imagery. Remote Sensing, 5(5), 2292-2307.

Woebbecke, D. M., Meyer, G. E., Von Bargen, K., \& Mortensen, D. A. (1995). Color indices for weed identification under various soil, residue, and lighting conditions. Transactions of the ASAE, 38(1), 259-269.

Wood, J. D., 1996: The geomorphological characterisation of digital elevation models. Ph.D. thesis, University of Leicester, http://www.soi.city.ac.uk/ jwo/phd. 